\title{
Automatic Arabic License Plate Recognition
}

\author{
Yasser M. Alginahi, Member, IACSIT
}

\begin{abstract}
Automatic License Plate (LP) recognition uses optical character recognition to read LPs on vehicles, such system is essential for traffic control, theft control, electronic toll collection, parking lots access and/or billing. The proposed Arabic LP recognition system is applied to Saudi Arabian LPs which have two different formats. Therefore, this system recognizes both Arabic and Indian numerals as well as limited Arabic and Latin alphabets. The system goes through many preprocessing steps in order to produce segmented characters of the LPs images. The feature extraction step uses the count of black pixels from the horizontal projection profiles in addition to the black pixel distributions in divided zones of the character image. The recognition is performed separately based on different regions; therefore, applying the recognition process to specific regions of the LPs; speeds up the processing due to the limited number of comparisons. The recognition step uses both a distance classifier and Neural Network (NN) classifier to discriminate between the different characters. This proposed system provides more importance to the preprocessing stage whose success guarantees the successful performance of the whole system. Also, the Arabic characters are verified against the corresponding Latin characters and similarly the Arabic numerals to their corresponding Indian numerals. The system is tested on $\mathbf{4 6 2}$ correctly localized LPs providing a $98.63 \%$ character recognition rate and a total of 94.9\% accepted LPs.
\end{abstract}

Index Terms-Arabic license plate, recognition, projection profile, feature extraction, Mahalanobis distance, Neural Networks (NNs).

\section{INTRODUCTION}

Automatic number plate recognition was invented in 1976 and the first working prototype system was implemented in 1979 in the UK [1]. LP recognition is used in many applications such as access control, traffic violations, law enforcements, border control, electronic billing ... etc., such systems are based on image processing and computer vision techniques. Several recognition techniques have been applied to Automatic LPs recognition systems these are: template matching [2]-[3], structural and statistical analysis [4], fuzzy logic [5] and NNs approaches [6].

All LPs found in Arab countries contain the name or abbreviation of the name of the country in Arabic or Latin. The rest of the LP may contain characters from Arabic alphabets, Indian numerals or characters from both Arabic and Latin alphabets as well as Arabic and /or Indian numerals depending on the country. LP systems related to Saudi Arabia have been proposed and developed in [2][3][6]. The systems developed are used on the old LPs only, more details are provided in section 3 on the Saudi LPs, the

Manuscript received January 18, 2011; revised April 24, 2011.

Yasser M. Alginahi is with Department of Computer Science College of Computer Science and Engineering, Taibah University, P.O. Box 344, Postal Code: 41411, Madinah, Saudi Arabia (email: yginahi@taibahu.edu.sa, alginahi@gmail.com). old kind contains 3 Arabic alphabets and 3 Indian numerals. The work of Sarfraz, et al. in 2003, [2], is the first attempt towards the recognition of Saudi Arabian LPs. The system extracts the LP from the captured image by detecting vertical edges using Sobel edge detector then the LP region is confirmed by comparing the detected region to the actual standard size ratio of the LP. In case of more than one region is detected as probable candidates for $\mathrm{LP}$ the $\mathrm{B} / \mathrm{W}$ ratio is tested which is determined according to a specific threshold calculated experimentally. Next, the LPs are divided into 6 regions, one region for each character or numeral, and vertical projections are calculated then the number of black pixels in each column are taken as the features to be extracted after normalizing each character to an image of 40x40. Finally, template matching based on the Hamming distance approach is used to match all the normalized characters to the characters in the database. The system provides a $95.24 \%$ recognition rate when tested on 610 LPs and the failure of some LPs is mainly due to the bad quality of the captured images which in turn causes wrong detection or extraction of vertical edges. The same exact work is also presented in [3] by Ahmed et al, which also contains the name of one of the authors in [2]. This system is tested on 710 LPs providing a $96.05 \%$ recognition rate [3].

The LP system for Saudi Arabia presented in [6] explains a similar system to [2][3] for all the stages of a LP system except the feature extraction and recognition stages. The feature extraction is based on the binary values of each character normalized to a size of $30 \mathrm{x} 40$ and the character recognition stage uses NNs instead of template matching Hamming distance approach. This system is in its initial stages and its objective is to develop a unified system for the Arab states of Gulf Cooperation Council, GCC, it is tested only on 61 Saudi LPs and the authors claim 97\% recognition rate. The authors did not present or explain how to recognize the LPs of different countries but instead presented a system intended for Saudi LPs. It is noticed that the work in [2]-[3] and [6] were all carried out at King Fahd University, Saudi Arabia.

The LPs for GCC are not of the same format and developing a unified system will need a sophisticated system that deals with all different kinds of fonts and LP designs. To develop a standard system for all Middle Eastern countries the structure/format of each country must be taken into consideration. Most of Arab countries LPs contain a combination from the following: Arabic alphabets, Latin alphabets, Arabic numerals, Indian numerals, name of country or its abbreviation in Arabic and/or Latin. The design of such a system is a major project that needs the cooperation between the research institute which is going to develop this system and all the governments involved, therefore, this is a large-scaled project which needs a large database of LP images from all different countries as well 
sufficient time and large man-power to be implemented and tested. For those interested in looking at LPs from different countries of the world, www.worldlicenseplates.com provides images of different versions and formats of license plates from almost all countries of the world.

The most recent work on Saudi LPs recognition is developed by Khalil, it uses the template matching technique for the recognition of both Saudi and Egyptian LPs; however, it is based on old Saudi LPs [7] and Egyptian LPs that follow the same format as the old Saudi LPs; however, it ignores other LP formats found in both countries. It uses similar techniques for all stages of the LP recognition system found in other published systems with the difference in the recognition stage only which uses a moving window template matching classifier developed in [8]. Surprisingly, this recent work did not cite any of the work published on Saudi LP recognition systems.

Other work found in literature for LP recognition of Middle Eastern countries includes: Egyptian LPs [9]-[10], Iranian LPs [11]-[12] and Tunisian LPs [13]. There are many other systems developed for automatic LPs recognition of other countries. The work in [14] presents an automatic license plate system which can be implemented in many countries which use Latin characters and Arabic numerals; the system is implemented using two different NN classifiers: the Multilayer Perceptron (MLP) NN and the Hopfield network. The performance of the system using the Hopfield network is $87 \%$ which on the other hand outperformed the MLP NN producing $80 \%$ recognition rate. There are many other systems which are developed for specific countries, for example, the system in [15] presents a system for Korean LP recognition based on edge matching. Another example is a LP recognition system for Malaysian LPs which uses NNs in the recognition process [16].

Most of the systems developed for Saudi Arabian and other country's LP systems use template matching. This technique requires fixed size LPs and same pixel intensity; therefore, such a technique cannot be used in real time applications due to the different lighting conditions under which LPs are captured, however, techniques based on NNs, statistical and distance classifiers are more suitable for such application.

The paper is organized as follows: section 2 presents the main stages of a typical LP system, section 3 provides an overview on Saudi Arabian LPs, Section 4 introduces the proposed LP recognition system, section 5 demonstrates the results obtained and finally section 6 concludes the paper.

\section{TYPICAL LP SYSTEMS}

A typical LP automatic recognition system goes through several major stages as shown in Figure 1 and each stage may contain several steps. The image acquisition can be done using a digital camera or a video camera with a frame grabber to select a frame. Following image capture, some preprocessing is required to prepare the image of the LP for the LP extraction stage. Next, the characters are detected then segmented and features are extracted from each character. Finally, the characters go through the recognition stage.

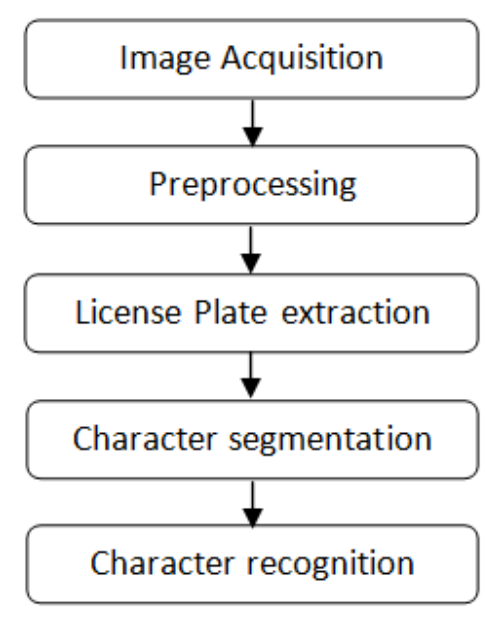

Figure 1.Steps of a typical LP system

\section{SAUDI LPS}

The Saudi LPs in circulation are of two kinds. The old kind includes 3 regions the top part contains Region 1 (R1) with the name of the country in Arabic, السعودية, and the lower part contains two regions, Region 2 (R2) has three Arabic alphabets and Region 3 (R3) has three Indian numerals, Figures 2 and 3.

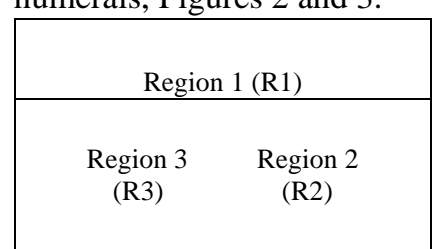

(a)

\begin{tabular}{|ccc|}
\hline Region 3 & Region 1 & Region 2 \\
(R3) & (R1) & (R2) \\
\hline
\end{tabular}

(c)

\begin{tabular}{|c|c|c|}
\hline Region 3 (R3) & Region 2 (R2) & 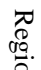 \\
\hline Region 5 (R5) & Region 4 (R4) & $\gtrsim$ \\
\hline
\end{tabular}

(d)

Figure 2. Block diagram of (a) Old Saudi LP (b) New Saudi LP (c) Wide old Saudi LP (d) Wide new Saudi LP (Figure not to scale).

The old LPs have different colors according to the purpose of use of the vehicle: white for private vehicles, green for diplomatic cars, yellow for public transports (taxicabs), blue for private transports, and grey for export and customs vehicles. On the other hand, the new LPs are mainly white, but include a vertical colored region on the right side, R1, containing the symbol of the kingdom as well as the letters KSA (abbreviating the name of the country, Kingdom of Saudi Arabia). The color indicates the intended use of the vehicle, same colors as presented above [17]. The rest of the new LP is divided into four regions two on top and two on bottom, Figures 2 and 3. The top right region, R2, contains three Arabic alphabets and the left top region R3, contains Arabic numbers where the numbers can have up to 4 digits. The bottom regions, R4 and R5, contain the corresponding Latin characters (in capital form only) and Indian numerals respectively according to Table 1. Figure 3 
shows examples of the two kinds of LPs. All characters are black for both kinds of LPs. To the best knowledge of the author, the old LPs will probably be eliminated by 2012 .

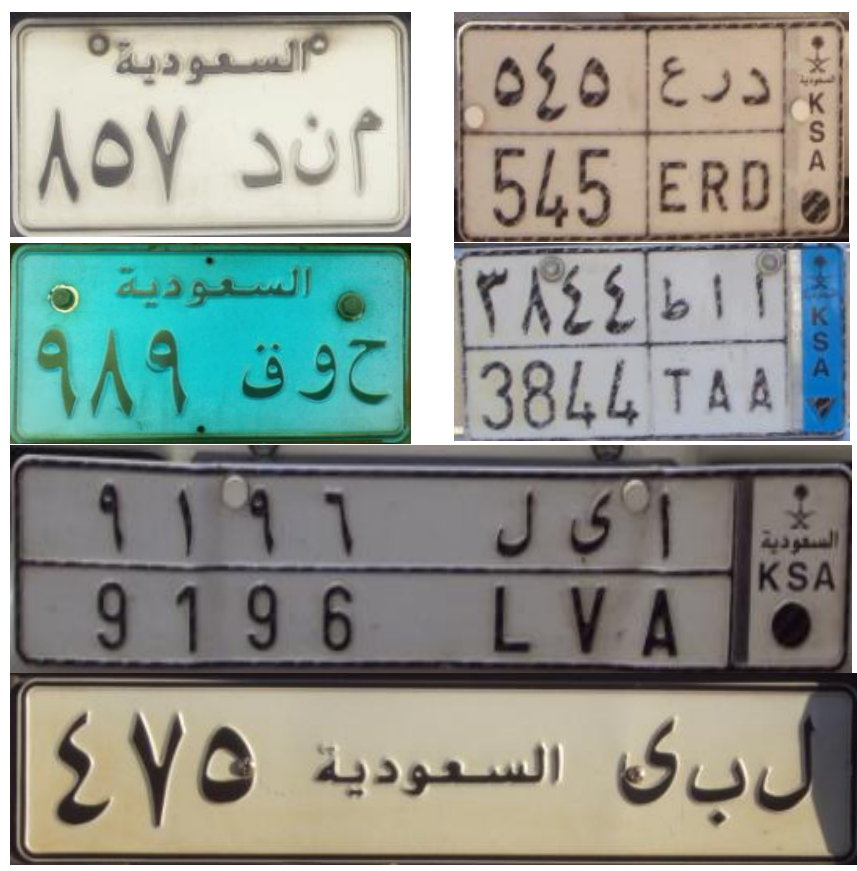

Figure 3. Examples of old and new Saudi LPs

TABLE 1. CHARACTERS USED IN SAUDI LP

\begin{tabular}{|c|c|}
\hline $\begin{array}{c}\text { Arabic } \\
\text { Alphabets }\end{array}$ & $\begin{array}{l}\text { Corresponding } \\
\text { Latin Characters }\end{array}$ \\
\hline 1 & $A$ \\
\hline ب & $B$ \\
\hline$\tau$ & $\mathrm{J}$ \\
\hline 2 & $\mathrm{D}$ \\
\hline J & $\mathrm{R}$ \\
\hline س & $\mathrm{S}$ \\
\hline ص & $X$ \\
\hline b & $\mathrm{T}$ \\
\hline$\varepsilon$ & $\mathrm{E}$ \\
\hline ث & $\mathrm{V}$ \\
\hline ق & $\mathrm{G}$ \\
\hline ك & $\mathrm{K}$ \\
\hline J & $\mathrm{L}$ \\
\hline r & Z \\
\hline ن ن & $\mathrm{N}$ \\
\hline$\Delta$ & $\mathrm{H}$ \\
\hline 9 & $\mathrm{U}$ \\
\hline
\end{tabular}

\begin{tabular}{|c|c|}
\hline $\begin{array}{c}\text { Arabic } \\
\text { Numerals }\end{array}$ & $\begin{array}{c}\text { Corresponding } \\
\text { Indian } \\
\text { Numerals }\end{array}$ \\
\hline 0 & $\cdot$ \\
\hline 1 & $\checkmark$ \\
\hline 2 & $r$ \\
\hline 3 & $r$ \\
\hline 4 & $\varepsilon$ \\
\hline 5 & $\bullet$ \\
\hline 6 & 7 \\
\hline 7 & $V$ \\
\hline 8 & $\wedge$ \\
\hline 9 & 9 \\
\hline
\end{tabular}

The two formats of LPs have wider versions with shorter heights, as shown in Figure 3. The old wide LP has a slightly different format than the regular old LP, the name of the country in Arabic, السعودية, is written in the middle of the LP between the 3 Arabic alphabets and 3 Indian numbers, Figure 3. On the other hand, the wider new LPs have the same format as the regular new LPs. The H:W ratio for the old LPs is approximately $1: 2$ and for the new LPs is approximately 1:4.5.

\section{Proposed LP RECOGNITION SySteM}

The proposed automatic LP recognition system for Saudi Arabian LPs goes through the following main stages: image acquisition, preprocessing, character segmentation, feature extraction and recognition. The proposed LP recognition system is shown in Figure 4. The details of each stage are presented in the following subsections.

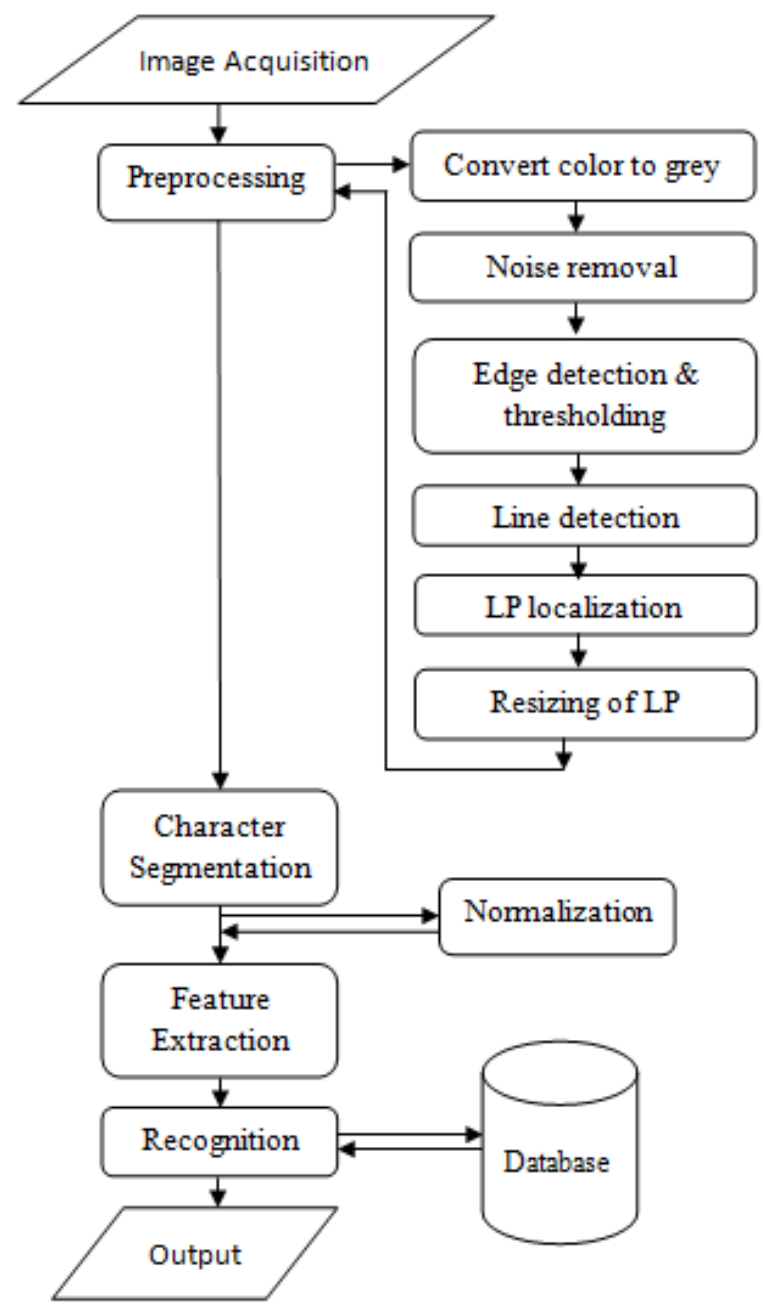

Figure 4, Arabic LP recognition system

\section{A. Image acquisition and preprocessing}

The LP images are captured in outdoor environment using a digital camera then the total intensity of the three primary colors is averaged to provide a grey-level image. Median filtering is used to remove noise because of its effectiveness in removing random occurrences of black and white pixels. Many other preprocessing techniques may also be used depending on image quality, for further information on preprocessing techniques the reader is referred to [18]. Following the noise removal step, the Sobel edge detection operator is applied to the image and within this step the image is converted into a binary image using the wellknown Otsu global thresholding method [19]. Vertical lines are then searched and for old LPs if two parallel lines are found the LP is detected then by connecting the ends of these two lines horizontally a rectangle is formed enclosing the LP. However, for the old and new LPs if more than two vertical lines are detected the width to height ratio of the LPs is calculated in order to locate the LP.

\section{B. Character segmentation}

The LP localization step identifies the complete area of the specific LP (old/new). The next step, if the LP is of the regular old kind, horizontal projection is performed in order to remove the upper part containing the word, السعودية, 
following this step, the 8-connected components technique is applied to find all the components (characters/numerals) on the image. The dimensions of each character are calculated so that to remove any unwanted objects due to noise, dirt or screw tops as well as dots on Arabic characters are removed from R2. Removing the dots will not affect the recognition of the shape of the character since there are only three characters containing dots, these are: ب، ن، ق, as shown in Table 1, and their shape is distinguishable without the dots. This step is also performed on R3 of the new kind of LPs even though the (Indian numeral zero $(\cdot)$ ) has the same size as dots found on some Arabic alphabets or the same size of a screw top, but to avoid the removal of the Indian zero numeral, the position of the numeral zero must be in a central location compared to the height of other numerals. For the old LPs the number of connected components in R2 and R3 are 3 in each region and for the new LPs the number of characters in R2 and R4 are compared to verify they are the same and equal to 3 , if there is any discrepancy then the components with small dimensions are eliminated during processing, similarly for R3 and R5 of the new LPs. Therefore, R2 = R4 = 3 characters and $(\mathrm{R} 3=\mathrm{R} 5) \leq 4$ numerals. After the character segmentation stage, the characters are normalized since the distance from the digital camera to the LP is not fixed. Each character is normalized to a size of $40 \times 30$ which is decided experimentally, the height is taken to be more than the width due to the fact that the Arabic alphabet used on LPs are taller compared to their width. The same procedure is repeated if the LP is detected to be of the new kind except that each region is processed separately and R1 is not considered during processing.

\section{Feature extraction}

Horizontal projection profiles as well as zoning are used as features extracted from each character. The horizontal projection profile provides 40 features for each character where black pixel count in each row is taken as a feature. The characters are also divided into zones of $5 \times 5$ where the black pixel distribution is also calculated from each zone to provide 48 features. In all, 88 features are extracted from each character. These features are then fed into the next step of the LP recognition system. Figure 5 shows an example on how the features are calculated from an Arabic character and Table 2 shows the 88 features of the example character $\Delta$.

\section{Recognition and interpretation}

The recognition step assigns a label to a character based on the information provided by its extracted features. The first step in this process is the training step where several sets of characters containing all the available alphanumeric characters are extracted from different LPs after they go through the preprocessing and character segmentation stages. Figure 6 shows an example of a complete set of all the alphanumeric characters used in the training stage of the Arabic LPs recognition system. Then the characters are normalized to a size of $40 \times 30$, features are extracted and saved in a database to be used during the recognition stage. These features are saved as the prototype features to be compared against during the recognition stage of the LP system.

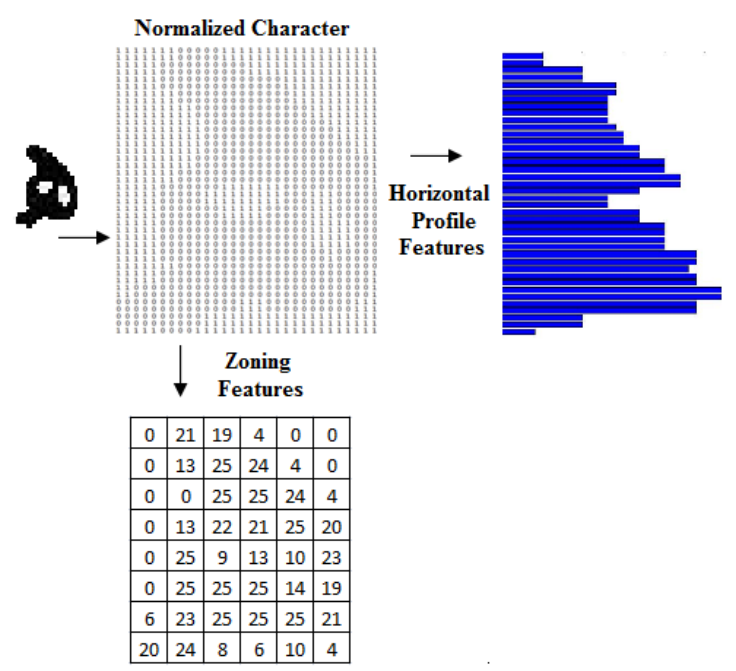

Figure 5. Horizontal profiles and divided zones for the character ( $\diamond)$.

TABLE 2. FEATURES EXTRACTED FOR THE EXAMPLE IN FIGURE 5. Horizontal Profile features: (40 features)

$5,5,10,10,14,14,13,13,13,13,14,15,15,17,17,20,20,22,22$,

$17,13,13,17,17,20,20,20,20,24,24,23,24,24,27,27,24,24,10$,

10,4

Zoning Features: (48 features)

$0,21,19,4,0,0,0,13,25,24,4,0,0,0,25,25,24,4,0,13,22,21$,

$25,20,0,25,9,13,10,23,0,25,25,25,14,19,6,23,25,25,25,21$,

$20,24,8,6,10,4$

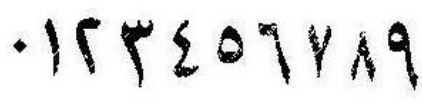

0123456789

\section{ABJORSXTEVGKLZNHU و هن}

Figure 6. A complete training set including all alphanumeric characters used in Saudi LPs.

The features extracted from each segmented character are: the normalized horizontal projection features (the pixel count from the horizontal projection profiles divided by the width of the normalized character, 40) and the black pixel count from the divided zones which are divided by 25 to provide normalized features. Therefore, the total number of features for each character is saved in a vector containing 88 values between 0 and 1 . Table 3 shows the normalized feature vector for the example shown in Figures 5, here, the feature vector shows the features separately just for clarity, otherwise, the features are stored together each in a feature vector containing 88 features.

TABLE 3. NORMALIZED FEATURES FOR EXAMPLE IN FIGURE 5. Normalized Horizontal Profile features: (40 features)

\begin{tabular}{|c|}
\hline 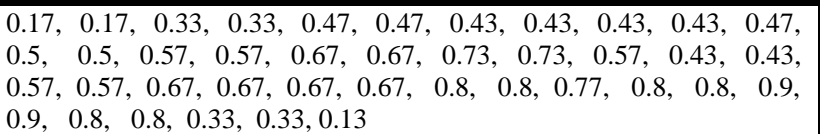 \\
\hline Normalized Zoning Feature \\
\hline $\begin{array}{l}0,0.84,0.76,0 . \\
0,0.52,0.88,0 .\end{array}$ \\
\hline
\end{tabular}


In this work, the Mahalanobis distance classifier, [20], is used to find the close match of the unknown character feature vector to one of the prototype characters whose feature vector are stored in the database. In the training stage, in order to speed up processing the types of characters are separated into four types according to the different regions on the LPs these are: Arabic numerals, Indian numerals, Arabic alphabets and Latin alphabets used in Arabic LPs. The Mahalanobis distance is based on correlations between variables by which different patterns can be identified and analyzed. It is a useful way of determining similarity of an unknown sample set to a known one. If there are two sets of vectors $x_{i}$ and $x_{j}$, each containing the same number of features, with means $\bar{x}_{i}$ and $\bar{x}_{j}$. Then the Mahalanobis distance is given by the following formula

$$
d_{i j}=\left(\left(\bar{x}_{i}-\bar{x}_{j}\right)^{T} S^{-1}\left(\bar{x}_{i}-\bar{x}_{j}\right)\right)^{\frac{1}{2}}
$$

where, $\mathrm{S}$ is the covariance matrix of the two feature vectors.

The feature vector of the unknown character is then compared to all sets of prototype vectors of the known characters/numerals in the database. The training of the LP system for each region is done separately, here, different training sets are used depending on the region of the LP in order to limit the number of comparisons. For example, characters in R2 of the old LPs are compared only to feature vectors of Arabic alphabets stored in the database and R3 is compared to Indian numerals only. Similarly, for the new LPs, in R2 only Arabic alphabets are compared, R3 only Indian numerals are compared, R4 only Latin alphabets are compared and in region 5 only Arabic numerals are compared. During the recognition stage the characters and their corresponding characters, shown in Table 1, are given the same class number which is used as a way of verification, but when displayed the characters are displayed according to the region they belong to. Therefore, when processed the character with feature vector providing the highest similarity measure to one of the prototype feature vectors in the database is taken as the candidate character which means that there is a very close similarity. On the other hand, if the character and its corresponding character from the other region are compared with no match then the character is reprocessed using a feed-forward multilayer NN (MLP-NN) classifier, [21], i.e., to confirm or reject the results obtained from the distance classifier, Figure 7. The MLP-NN is trained by supervised learning using the iterative backpropagation algorithm. In the learning phase, a set of input patterns, training set, are presented at the input layer as feature vectors, for example Table 3 provides a feature vector for the letter $(\diamond)$ which contains 88 features, together with their corresponding desired output pattern, which usually represents the classification for the input pattern. A feature vector is obtained for all the characters in the training sets. An example of a complete training set is shown in Figure 6, a total of ten complete training sets were used. Training the NN starts with small random weights, for each input pattern the network is required to adjust the weights attached to the connections so that the difference between the network's output and the desired output for that input pattern is decreased. Finally, the weights are calculated to be used during the classification/recognition stage. Four different weights were obtained depending on the types of characters in order to speed up the processing. During classification, the extracted features are fed into the network that performs classification by assigning a class number to each block segment using the binary values computed at the output layer. Finally, the unknown characters are recognized.

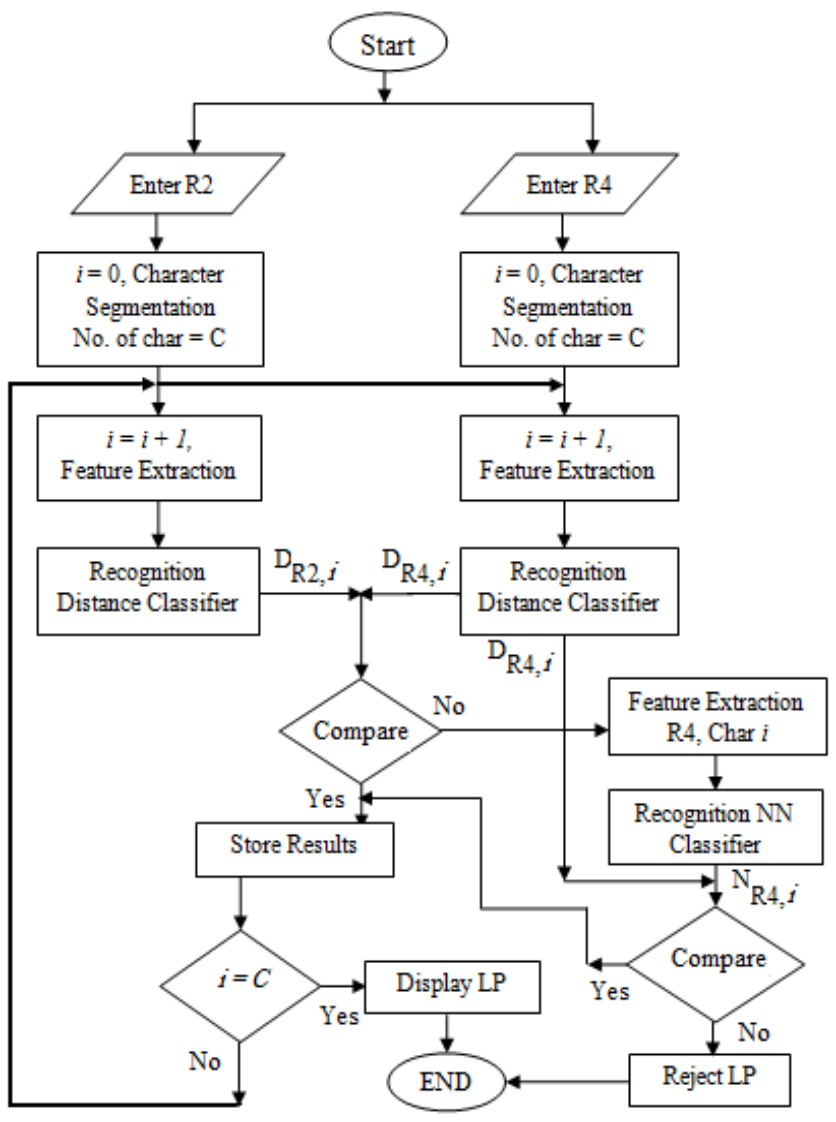

Figure 7. The recognition process for new type LPs.

From Figure 7, for the new LPs, this means if the character in R2 and its corresponding character in R4 don't match as shown in Table 1an extra step is required to verify if using a different classifier will produce a match either with the character in R2 or R4. Figure 7, shows an example where if the output of the distance classifier $D_{R 2, i}$ does not match with $D_{R 4, i}$ then the character is passed through the $\mathrm{NN}$ classifier to check if it matches i.e., $D_{R 4, i}$ is compared to $\mathrm{N}_{\mathrm{R} 4, \mathrm{i}}$.

In this system if the result of the $\mathrm{NN}$ matches with the Mahalanobis distance classifier then the result is confirmed and the character is recognized, otherwise, the character is rejected and the LP is processed manually. Similarly, if the preprocessing stage fails to locate the LP due to bad quality image, noise or dirt residue on the plate; the plate is rejected and then processed manually. The mismatch of even one character during the comparison process will result in the LP being rejected. Figure 8 shows the program interface with an example of a LP processed correctly. 


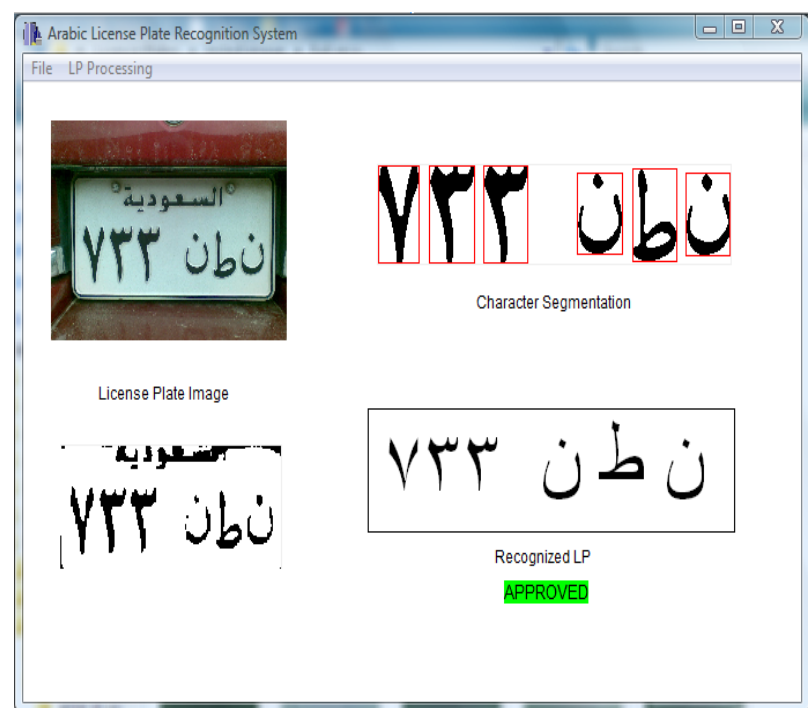

Figure 8 shows the GUI of the software with an example of a rejected LP.

\section{RESULTS AND OBSERVATIONS}

The system is tested on 470 LP images captured in outdoor environment. The preprocessing stage presents a success rate of $98.3 \%$ correctly localized LPs. All LPs passing the preprocessing stage contain a total number of characters equal to 4392 from both LP types. 60 characters are classified as rejected when passed through the LP recognition system which corresponds to $98.63 \%$ character recognition, however, in case there is a doubt in recognizing any character then it is rejected and a flag is set to process the whole LP manually. The results are tabulated in Table 4. The overall number of accepted LPs after deducting the unsuccessful LPs during both the localization and recognition steps is calculated as $94.9 \%$.

TABLE 4. RECOGNITION RESULTS FOR THE PROPOSED ARABIC LP RECOGNITION SYSTEM.

\begin{tabular}{|l|c|c|c|}
\hline & Old LPs & $\begin{array}{c}\text { New } \\
\text { LPs }\end{array}$ & Totals \\
\hline No. of LPs (T) & 250 & 220 & 470 \\
\hline $\begin{array}{l}\text { Unsuccessful LP localization } \\
\text { (UL) }\end{array}$ & 3 & 5 & 8 \\
\hline LP correctly localized & 247 & 215 & 462 \\
\hline Total No. of characters & 1482 & 2910 & 4392 \\
\hline No. of recognized characters & 1464 & 2868 & 4332 \\
\hline \% of recognized characters & $98.79 \%$ & $98.56 \%$ & $98.63 \%$ \\
\hline No. of rejected characters & 18 & 42 & 60 \\
\hline $\begin{array}{l}\text { Number of LP affected by the } \\
\text { rejected characters (UR) }\end{array}$ & 7 & 9 & 16 \\
\hline$\%$ of rejected characters & $1.21 \%$ & $1.44 \%$ & $1.37 \%$ \\
\hline $\begin{array}{l}\text { Total accepted LPs } \\
=\mathrm{T}-\text { UL - UR }\end{array}$ & 240 & 206 & 446 \\
\end{tabular}

\section{CONCLUSION}

The proposed research work is the first of its kind for Saudi Arabian new license plates which also includes old type of LPs. The proposed system is tested over a large image dataset captured in various illumination conditions and is verified to generate high recognition rate. This work is low cost and saves time for improving security when used by law enforcement and private organizations. The proposed LP recognition system for Saudi old and new LPs is implemented using both a distance and NN classifiers.
The Mahalanobis Distance measure is used to measure the similarity between the characters tested and the prototype ones. On the other hand, the MLP-NN classifier is used to verify or reject the results. The successful of this system is credited to the preprocessing stage whose success provides accurate detection of LPs. The system is applied to 470 Saudi LPs (old and new) where eight LPs were rejected because of unsuccessful LP localization and therefore from the 462 successfully localized LPs 4392 characters are extracted and the system provides a total character recognition rate of $98.63 \%$ not including the initially rejected LPs in the preprocessing stage. Finally, the total rejected LPs including those from the preprocessing stage are 24 from a total of 470 LPs used in this system.

\section{REFERENCES}

[1] <http://en.wikipedia.org/wiki/Automatic_number_plate_recognition>, accessed June 20, 2010.

[2] M. Sarfraz, M. J,. Ahmed and S. A. Ghazi, "Saudi Arabian License Plate Recognition System," International Conference on Geometric Modeling and Graphics, pp. 36-41, 2003.

[3] M. J. Ahmed, M. Sarfraz, A. Zidouri, and W. G. A Khatib, "License Plate Recognition System," $10^{\text {th }}$ IEEE International Conference on Electronics, Circuits and Systems,, pp. 898-901, 2003.

[4] Xin Fan Guoliang Fan Dequn Liang, "Joint Segmentation and Recognition of License Plate Characters," IEEE International Conference on Image Processing, Vol. 4, pp. IV 353 - IV 356, 2007.

[5] Nijhuis, J.A.G.; Ter Brugge, M.H.; Helmholt, K.A.; Pluim, J.P.W.; Spaanenburg, L.; Venema, R.S. and Westenberg, M.A., "Car License Plate recognition with neural networks and fuzzy logic," IEEE International Conference on Neural Networks, Vol.5, pp. 2232 $-2236,1995$

[6] A. Zidouri and M. Deriche, "Recognition of Arabic License Plates Using NN," First workshops on image processing theory tools and applications, pp. 1-4, 2008

[7] M. I. Khalil, "Car Plate Recognition Using the Template Matching Method," International Journal of Computer Theory and Engineering, Vol. 2, No. 5, pp. 683- 687, 2010.

[8] M. C. Fairhurst and M. S. Hoque, Moving Window Classifier: a new approach to off-line image recognition Electronic Letters, Vol. 37, No. 7, pp. $381-392,1996$

[9] A. A. Sewisy, "Arabic License Plate Recognition Based on Hough Transform," ICGST Artificial Intelligence and Machine Learning $\begin{array}{llllll}\text { Journal, Vol. 7, No. 1, pp. } & 1-7, \quad 2009 .\end{array}$ <www.icgst.com/aim1/Volume7/Issue1/P112065 0001. pdf>

[10] M. El-Adawi, H. A. Keshk and M. M. Horagi, "Automatic LP Recognition," "........

[11] Delforouzi, A.; Pooyan, M.; "Efficient Farsi LP recognition," $7^{\text {th }}$ International Conference on Information, Communications and Signal Processing, pp. 1-5, 2009.

[12] A. Broumandnia and M. Fathy, "Application of Pattern Recognition for Farsi License Plate Recognition," ICGST International Conference on Graphics, Vision, and Image Processing, ICGSTGVIP Journal, Vol. 5, No. 2, pp. 25 - 31, Dec. 2005.

[13] Ameur CHHAYDER et Imene BELHADJ MOHAMED, "Système de Reconnaissance Automatique des Plaques Minéralogiques," International Conference: Sciences of Electronic, Technologies of Information and Telecommunications, pp. 1 - 8, March 22-26, 2009 - Tunisia.

[14] A. Akoum, B. Daya and P. Chauvet, "Two Neural Networks for License Number Plates Recognition," Journal of Theoretical and Applied Information Technology, Vol. 12, pp. 25 - 32, 2010.

[15] M., Yu, and Y.D. Kim, 2000. "An approach to Korean license plate recognition based on vertical edge matching", IEEE International Conference on Systems, Man, and Cybernetics, vol. 4, pp. 2975-2980.

[16] O. Khalifa, S. Khan, R. Islam and A. Suleiman, "Malaysian Vehicle License Plate Recognition," The International Arab Journal of Information Technology, Vol. 4, No. 4, pp. 359 - 364, October 2007.

[17] <http://www.saudiinfocus.com/en/forum/showthread.php?t=2074>, accessed July 06, 2010.

[18] Yasser Alginahi. Chapter 1: Preprocessing Techniques in Character Recognition, Character Recognition, Edited by Minoru Mori, ISBN: 978-953-307-105-3, Sciyo, 2010. 
[19] Otsu, N., "A threshold Selection Method from Grey-level Histograms," IEEE Transactions on Systems, Man and Cybernetics, SMC-9, pp. 6266, 1979.

[20] <http://people.revoledu.com/kardi/tutorial/Similarity/MahalanobisDis tance.html>, accessed August 23, 2010.

[21] M. A. Sid-Ahmed, Image Processing: Theory, Algorithms, and Architectures, McGraw-Hill, New York, NY, 1995.

Yasser M. Alginahi, Senior IEEE member since 2010, earned a Ph.D., in electrical engineering from the University of Windsor, Canada, a Master of
Science in electrical engineering and a Bachelors of Science in biomedica engineering from Wright State University, Ohio, U.S.A. Currently, he is an Assistant Professor, Dept. of Computer Science, College of Computer Science and Engineering, Taibah University, Madinah, KSA. His curren research interests are Pattern Recognition (OCR, Face recognition), crowd/traffic management and e-learning technologies. He is a licensed Professional Engineer and a member of Professional Engineers Ontario, Canada (PEO). He has executed several scientific projects in the field of pattern recognition and crowd/traffic management. He is editorial board member of several international journals and served as reviewer and member of scientific committees in several conferences. He published book entitled "Document Image Analysis" and has over 30 research publications and technical reports to his credit. 